\title{
SOME APPLICATIONS OF HOMOGENEOUS DYNAMICS TO NUMBER THEORY
}

\author{
DMitry KLeinbock
}

This survey paper is not a complete reference guide to number-theoretical applications of ergodic theory. Instead, the plan is to consider an approach to a class of problems involving Diophantine properties of $n$-tuples of real numbers, namely, describe a specific dynamical system which is naturally connected with these problems.

\section{A glimpse at Diophantine approximation}

For motivation, let us start by looking at two (vaguely defined) Diophantine problems:

Problem 1. Given a nondegenerate indefinite quadratic form of signature $(m, n)$, study the set of its values at integer points.

Here is a precise statement along these lines, conjectured in 1929 by Oppenheim [Op1] and proved in 1986 by Margulis [Ma3]:

Theorem 1.1. Let $B$ be a real nondegenerate indefinite quadratic form of signature $(m, n), k=m+n>2$. Then either $B$ is proportional to a rational form, or $\inf _{\mathbf{x} \in \mathbb{Z}^{k} \backslash\{0\}}|B(\mathbf{x})|=0$.

One possible approach to the problem is to write $B(\mathbf{x})=\lambda S_{m, n}(g \mathbf{x})$, where $\lambda \in \mathbb{R}, g \in S L_{k}(\mathbb{R})$ and

$$
S_{m, n}\left(x_{1}, \ldots, x_{k}\right)=x_{1}^{2}+\cdots+x_{m}^{2}-x_{m+1}^{2}-\cdots-x_{k}^{2}
$$

(a linear unimodular change of variables). Then the problem reduces to studying values of the standard form $S_{m, n}$ of signature $(m, n)$ applied to the collection of vectors of the form $\left\{g \mathbf{x} \mid \mathbf{x} \in \mathbb{Z}^{k}\right\}$. And the dynamical approach consists of studying the action of the stabilizer of the form $S_{m, n}$ on such collections.

Problem 2. Given $m$ vectors $\mathbf{y}_{1}, \ldots, \mathbf{y}_{m} \in \mathbb{R}^{n}$ (viewed as linear forms $\mathbf{x} \mapsto \mathbf{y}_{i} \cdot \mathbf{x}$, $\mathbf{x} \in \mathbb{R}^{n}$ ) how small (simultaneously) can be the values of $\left|\mathbf{y}_{i} \cdot \mathbf{q}+p_{i}\right|, p_{i} \in \mathbb{Z}$, when $\mathbf{q}=\left(q_{1}, \ldots, q_{n}\right) \in \mathbb{Z}^{n}$ is far from 0 ?

Let us also illustrate this by a conjecture, this time still open. Here we specialize to the case of just one linear form given by $\mathbf{y} \in \mathbb{R}^{n}$. The following is known as Littlewood's (1930) Conjecture:

The author was supported in part by NSF Grants DMS-9704489 and DMS-0072565. 
Conjecture 1.2. For every $\mathbf{y} \in \mathbb{R}^{n}, n \geq 2$, one has

$$
\inf _{\mathbf{q} \in \mathbb{Z}^{n} \backslash\{0\}, p \in \mathbb{Z}}|\mathbf{y} \cdot \mathbf{q}+p| \cdot \Pi_{+}(\mathbf{q})=0,
$$

where $\Pi_{+}(\mathbf{q})$ is defined to be equal to $\prod_{i=1}^{n} \max \left(\left|q_{i}\right|, 1\right)$ or, equivalently, $\prod_{q_{i} \neq 0}\left|q_{i}\right|$.

To approach Problem 2, one can put together

$$
\mathbf{y}_{1} \cdot \mathbf{q}+p_{1}, \ldots, \mathbf{y}_{m} \cdot \mathbf{q}+p_{m} \text { and } q_{1}, \ldots, q_{n},
$$

and consider the lattice

$$
\left\{\left(\begin{array}{c}
Y \mathbf{q}+\mathbf{p} \\
\mathbf{q}
\end{array}\right) \mid \mathbf{p} \in \mathbb{Z}^{m}, \mathbf{q} \in \mathbb{Z}^{n}\right\}=L_{Y} \mathbb{Z}^{m+n},
$$

where

$$
L_{Y} \stackrel{\text { def }}{=}\left(\begin{array}{cc}
I_{m} & Y \\
0 & I_{n}
\end{array}\right)
$$

and $Y$ is the matrix with rows $\mathbf{y}_{1}^{T}, \ldots, \mathbf{y}_{m}^{T}$. In this case, the orbit of the lattice (1.2) under a certain group action provides a way to study Diophantine properties of $\mathbf{y}_{1}, \ldots, \mathbf{y}_{m}$.

In both cases, we use the initial data of a number-theoretic problem to construct a lattice in a Euclidean space, and then work with the collection of all such objects (lattices). Our goal is to describe several principles responsible for a particular class of applications of flows in the space of lattices to number theory. For more details and a broader picture the reader is referred to a number of extensive reviews of homogeneous actions and interactions with number theory which have appeared during the last 10 years, such as: ICM talks of Margulis [Ma5], Ratner [Ra3], Dani [D5] and Eskin [E], books [St2] and [BMa], and survey papers [D6, D7, KSS, Ma7, St1].

The structure of this paper is as follows: in the next section we collect all basic facts about the space of lattices, and discuss a "lattice" approach to studying values of quadratic forms at integer points (Problem 1 and Theorem 1.1 in particular). Then in $\S \S 3$ and 4 we take several sub-problems of Problem 2 and describe recent results obtained by means of homogeneous dynamics. The last section is devoted to Conjecture 1.2 and related issues, that is, so called "multiplicative Diophantine approximation".

\section{THE SPACE OF LATTICES}

Phase space. Fix $k \in \mathbb{N}$ and consider

$$
\Omega \stackrel{\text { def }}{=} \text { the set of unimodular lattices in } \mathbb{R}^{k}
$$

(discrete subgroups with covolume 1). That is, any lattice $\Lambda \in \Omega$ is equal to $\mathbb{Z} \mathbf{v}_{1} \oplus \cdots \oplus \mathbb{Z} \mathbf{v}_{k}$, where the set $\left\{\mathbf{v}_{1}, \ldots, \mathbf{v}_{k}\right\}$ (called a generating set of the lattice) is linearly independent, and the volume of the parallelepiped spanned by $\mathbf{v}_{1}, \ldots, \mathbf{v}_{k}$ is equal to 1 .

An element of $\Omega$ which is easy to distinguish is $\mathbb{Z}^{k}$ (the standard lattice). In fact, any $\Lambda \in \Omega$ is equal to $g \mathbb{Z}^{k}$ for some $g \in G \stackrel{\text { def }}{=} S L_{k}(\mathbb{R})$. That is, $G$ acts transitively on $\Omega$, and, further, $\Gamma \stackrel{\text { def }}{=} S L_{k}(\mathbb{Z})$ is the stabilizer of $\mathbb{Z}^{k}$. In other words, $\Omega$ is isomorphic to the homogeneous space $G / \Gamma$. 
Measure. One can consider a Haar measure on $G$ (both left and right invariant) and the corresponding left-invariant measure on $\Omega$. It is well known that the resulting measure happens to be finite. We denote by $\mu$ the normalized Haar measure on $\Omega$.

Topology. Two lattices are said to be close if generating sets which are close to each other can be chosen for them. This defines a topology on $\Omega$ which coincides with the quotient topology on $G / \Gamma$. An important feature is that $\Omega$ is not compact (in other words, $\Gamma$ is a non-uniform lattice in $G$ ). More precisely, one has

Theorem 2.1 (Mahler's Compactness Criterion, see $[\mathrm{R}]$ ). A subset $K$ of $\Omega$ is bounded iff there exists $\varepsilon>0$ such that for any $\Lambda \in K$ one has $\inf _{\mathbf{x} \in \Lambda \backslash\{0\}}\|\mathbf{x}\| \geq \varepsilon$. In other words, define

$$
\Omega_{\varepsilon} \stackrel{\text { def }}{=}\{\Lambda \in \Omega \mid\|\mathbf{x}\|<\varepsilon \text { for some } \mathbf{x} \in \Lambda \backslash\{0\}\} ;
$$

then $\Omega \backslash \Omega_{\varepsilon}$ is compact.

Action. $\Omega$ is a topological $G$-space, with the (continuous) left action defined by

$$
g \Lambda=\{g \mathbf{x} \mid \mathbf{x} \in \Lambda\} \quad \text { or } \quad g(h \Gamma)=(g h) \Gamma .
$$

One can consider the action of various subgroups (one- or multi-parameter) or subsets of $G$. Thus one gets an interesting class of dynamical systems. Several important features of these systems are worth mentioning.

First, the geometry of the phase space is "uniform": a small enough neighborhood of every point of $\Omega$ is isometric to a neighborhood of identity in $G$. In other words, many geometric constructions can be reduced to algebraic manipulations in $G$.

Second, the very rich representation theory of $G$ can be heavily used. Namely, the $G$-action on $\Omega$ can be studied via the regular representation of $G$ on $L^{2}(\Omega)$

The two features above in fact apply for all homogeneous actions, that is, actions of subgroups of a Lie group $G$ on the quotient space $G / \Gamma$ where $\Gamma$ is a lattice in $G$. There are also important features specifically for the space $\Omega=S L_{k}(\mathbb{R}) / S L_{k}(\mathbb{Z})$ : namely, combinatorial structure of the space of lattices, as well as intuition coming from the theory of Diophantine approximation.

In what follows we will focus our attention on the space $\Omega$, but most of the results will be valid in much bigger generality of homogeneous actions, which will be indicated. The reader is referred to [AGH, Ma6, R, St2, Z] for general facts about Lie groups, discrete subgroups and homogeneous spaces.

Classification of actions. Let $G$ be a Lie group and $\Gamma$ a discrete subgroup. Since $g(h \Lambda)=\left(g h g^{-1}\right) g \Lambda$ for every $\Lambda \in G / \Gamma$ and $g, h \in G$, local properties of the $g$-action are determined by the differential of the conjugation map, $\operatorname{Ad}_{g}(x)=$ $\left.\frac{d\left(g \exp (t x) g^{-1}\right)}{d t}\right|_{t=0}$ (here $x$ belongs to the Lie algebra of $G$ ). An element $g \in G$ is said to be: unipotent if $\left(\operatorname{Ad}_{g}-\mathrm{Id}\right)^{j}=0$ for some $j \in \mathbb{N}$ (equivalently, all eigenvalues of $\operatorname{Ad}_{g}$ are equal to 1); quasi-unipotent if all eigenvalues of $\mathrm{Ad}_{g}$ are of absolute value 1; partially hyperbolic if it is not quasi-unipotent.

Given $g \in G$, define

$$
H^{ \pm}(g)=\left\{h \in G \mid g^{-l} h g^{l} \rightarrow e \text { as } l \rightarrow \pm \infty\right\}
$$


(expanding and contracting horospherical subgroups). Then $G$ is locally a direct product of $H^{-}(g), H^{+}(g)$ and another subgroup $H^{0}(g)$, and $g$ is quasiunipotent iff $H^{0}(g)=G$ (that is, $H^{-}(g)$ and $H^{+}(g)$ are trivial). Furthermore, for any $\Lambda \in G / \Gamma$ the orbits $H^{-}(g) \Lambda, H^{+}(g) \Lambda$ and $H^{0}(g) \Lambda$ are leaves of stable, unstable and neutral foliations on $G / \Gamma$.

We now specialize to the case $G=S L_{k}(\mathbb{R})$ and $\Gamma=S L_{k}(\mathbb{Z})$.

Example. The simplest case is when $k=2$ : then $\Omega=G / \Gamma$ is isomorphic to the unit tangent bundle to the surface $\mathbb{H}^{2} / S L_{2}(\mathbb{Z})$. The geodesic flow is then given by the action of $\left(\begin{array}{cc}e^{t} & 0 \\ 0 & e^{-t}\end{array}\right)$, and the horocycle flow - by the action of $\left(\begin{array}{ll}1 & t \\ 0 & 1\end{array}\right)$ (the simplest example of a unipotent flow).

More examples. Suppose that $g \in G$ is diagonalizable over $\mathbb{R}$, and take a basis of $\mathbb{R}^{k}$ in which $g=\operatorname{diag}(\underbrace{\lambda_{1}, \ldots, \lambda_{1}}_{i_{1} \text { times }}, \ldots \ldots, \underbrace{\lambda_{l}, \ldots, \lambda_{l}}_{i_{l} \text { times }}), \quad \lambda_{1}>\cdots>\lambda_{l}$. Then $H^{-}(g)$ and $H^{+}(g)$ are subgroups of lower- and upper- triangular groups. An important special case occurs when $g$ as above comes from "the most singular" direction in a Weyl chamber of the Lie algebra of $G$; that is, when it has only two distinct eigenvalues. In this case one can write $k=m+n$ and consider a one-parameter subgroup of $G=S L_{k}(\mathbb{R})$ given by

$$
g_{t}=\operatorname{diag}\left(e^{t / m}, \ldots, e^{t / m}, e^{-t / n}, \ldots, e^{-t / n}\right) .
$$

Then the expanding horospherical subgroup of $G$ relative to $g_{1}$ is exactly $\left\{L_{Y}\right.$ | $\left.Y \in M_{m \times n}(\mathbb{R})\right\}$, where $L_{Y}$ is as defined in (1.3).

Ergodic properties. Here the main tool is the representation theory of semisimple Lie groups. By a theorem of Moore [Mo1], the action of any noncompact closed subgroup of $G$ on $\Omega=G / \Gamma$ is ergodic and, moreover, mixing; in other words, matrix coefficients $(g \varphi, \psi)$ of square-integrable functions on $\Omega$ with mean value zero tend to 0 as $g \rightarrow \infty$ in $G$. (Here $(\cdot, \cdot)$ stands for the inner product in $L^{2}(\Omega)$.) In fact for smooth functions this decay is exponential, as shown in the following

Theorem 2.2 (Decay of correlations). There exists $\beta>0$ such that for any two functions $\varphi, \psi \in C_{\text {comp }}^{\infty}(G / \Gamma)$ with $\int \varphi d \mu=\int \psi d \mu=0$ and any $g \in G$ one has

$$
\left|\int(g \varphi \cdot \psi) d \mu\right| \leq \operatorname{const}(\varphi, \psi) e^{-\beta\|g\|}
$$

In particular, if $g_{t}$ is partially hyperbolic, then

$$
\left|\int\left(g_{t} \varphi \cdot \psi\right) d \mu\right| \leq \operatorname{const}\left(\varphi, \psi, g_{t}\right) e^{-\gamma t}
$$

See [Mo2, Ra1] for $k=2$, [KS] for $k>2$.

The following result can be derived from the mixing property of partially hyperbolic actions on $G / \Gamma$ : 
Theorem 2.3 (Uniform distribution of unstable leaves, [KM1]). Let $g_{t}$ be a partially hyperbolic one-parameter subgroup of $G, H=H^{+}\left(g_{1}\right)$, $\nu$ a Haar measure on $H$. Then for any open subset $V$ of $H$, any $\varphi \in C_{\text {comp }}^{\infty}(G / \Gamma)$ and any compact subset $Q$ of $G / \Gamma$, the average of $\varphi$ over the $g_{t}$-image of $V \Lambda, \Lambda \in G / \Gamma$, tends to the integral of $\varphi$ as $t \rightarrow \infty$ uniformly (in $\Lambda$ ) on compact subsets of $G / \Gamma$; that is,

$$
\frac{1}{\nu\left(g_{t} V g_{-t}\right)} \int_{g_{t} V g_{-t}} \varphi\left(h g_{t} \Lambda\right) d \nu(h) \rightarrow \int_{G / \Gamma} \varphi d \mu .
$$

Remarks. Moore's theorem (that is, a criterion for mixing of subgroup actions) was proved under the assumption that

$$
\begin{aligned}
& G \text { is a connected semisimple Lie group with finite center } \\
& \text { and no compact factors, and } \Gamma \text { is an irreducible lattice in } G \text {, }
\end{aligned}
$$

(A lattice is irreducible if it is not, up to commensurability, a product of lattices in simple factors of $G$.) See also [BM, Ma5] for more general ergodicity and mixing criteria. Theorems 2.2 was proved in $[\mathrm{KS}]$ assuming (2.4) and in addition that

$$
\text { all simple factors of } G \text { have property (T). }
$$

One also knows, see [Bek, Lemma 3], that Theorem 2.2 holds when the group $G$ is simple. In [KM3] it was shown that one can remove condition (2.5) but instead assume that $\Gamma \subset G$ is a non-uniform lattice.

Theorem 2.3 is also proven in the generality of the assumption (2.4) (in fact, one only needs mixing of the $g_{t}$-action), and as long as Theorem 2.2 holds, the convergence in (2.3) is exponential in $t$. See [KM1, K4] for more details and generalizations, and [Ma7, Remark 3.10] for references to other related results and methods.

Recurrence of unipotent trajectories. In this subsection we are back to the case $G=S L_{k}(\mathbb{R})$ and $\Gamma=S L_{k}(\mathbb{Z})$. It is an elementary geometric observation that horocyclic trajectories on $S L_{2}(\mathbb{R}) / S L_{2}(\mathbb{Z})$ do not run off to infinity. It is much harder to prove that the same holds for any unipotent flow on $\Omega=S L_{k}(\mathbb{R}) / S L_{k}(\mathbb{Z})$ for $k \geq 3$ [Ma2, D3]. The theorem below, due to Dani (1985), is a quantitative strengthening; it shows that for any unipotent orbit one can find a compact subset of $\Omega$ such that the density of time that the orbit spends in this set is as close to 1 as one wishes:

Theorem 2.4 [D3]. For any $\Lambda \in \Omega$ and any $\delta>0$ there exists $\varepsilon>0$ such that for any unipotent subgroup $\left\{u_{x} \mid x \in \mathbb{R}\right\}$ of $G$ and any $T>0$ one has

$$
\left|\left\{x \in[0, T] \mid u_{x} \Lambda \in \Omega_{\varepsilon}\right\}\right| \leq \delta T .
$$

The proof is based on the combinatorial structure of the space of lattices. We will obtain the theorem above as a corollary of a more general fact in $\S 4$.

Orbit closures of unipotent flows. It has been proved by Hedlund that any orbit of the horocycle flow on $S L_{2}(\mathbb{R}) / S L_{2}(\mathbb{Z})$ is either periodic or dense. A farreaching generalization has been conjectured by Raghunathan and proved in full generality by Ratner. In particular, one has the following 
Theorem 2.5. [Ra2] Let $G$ be a connected Lie group, $\Gamma$ a lattice in $G$, and let $U$ be a subgroup of $G$ generated by unipotent one-parameter subgroups. Then for any $x \in G / \Gamma$ there exists a closed subgroup $L$ containing $U$ such that the closure of the orbit $U x$ coincides with $L x$ and there is an L-invariant probability measure supported on $L x$.

We emphasize that Theorem 2.5 allows one to understand all (not just almost all) orbits. It is this feature which is responsible for applications of this theorem to number theory. Let us illustrate it by sketching the reduction of the Oppenheim Conjecture (Theorem 1.1) to Ratner's theorem.

Corollary 2.6. Let $S\left(x_{1}, x_{2}, x_{3}\right)=2 x_{1} x_{3}-x_{2}^{2}$, and

$$
H_{S}=\left\{h \in S L_{3}(\mathbb{R}) \mid S(h \mathbf{x})=S(\mathbf{x}) \forall \mathbf{x} \in \mathbb{R}^{3}\right\} \cong S O(2,1)
$$

(the stabilizer of $S$ ). Then any relatively compact orbit $H_{S} \Lambda, \Lambda$ a lattice in $\mathbb{R}^{3}$, is compact.

Proof. $H_{S}$ is generated by its unipotent one-parameter subgroups, namely

$$
u(t)=\left(\begin{array}{ccc}
1 & t & t^{2} / 2 \\
0 & 1 & t \\
0 & 0 & 1
\end{array}\right) \text { and } u^{T}(t)=\left(\begin{array}{ccc}
1 & 0 & 0 \\
t & 1 & 0 \\
t^{2} / 2 & t & 1
\end{array}\right)
$$

and there are no intermediate subgroups between $H_{S}$ and $S L_{3}(\mathbb{R})$. Hence by Theorem 2.5 any $H_{S}$-orbit is either closed or dense.

The following is crucial for the deduction of the Oppenheim conjecture from the above corollary (implicitly stated in [CS] and later observed by Raghunathan):

Lemma 2.7. Let $B$ be a real nondegenerate indefinite quadratic form in 3 variables. Write $B(\mathbf{x})=\lambda S(g \mathbf{x})$ for some $g \in S L_{3}(\mathbb{R})$. Then the orbit $H_{S} g \mathbb{Z}^{3}$ is relatively compact if and only if

$$
|B(\mathbf{x})| \geq \varepsilon \text { for some } \varepsilon>0 \text { and all } \mathbf{x} \in \mathbb{Z}^{3} \backslash\{0\} .
$$

Proof. By transitivity of the action of the stabilizer $H_{B}$ of the form $B$ on the level sets of $B$ in $\mathbb{R}^{3} \backslash\{0\}$ and by continuity of $B$ at zero, assertion (2.6) is equivalent to the norm of $h \mathbf{x}$ being not less than $\varepsilon$ for some $\varepsilon>0$ and all $\mathbf{x} \in \mathbb{Z}^{3} \backslash\{0\}$ and $h \in H_{B}$. The latter, in view of Theorem 2.1, is equivalent to the orbit $H_{B} \mathbb{Z}^{3}$ being relatively compact in $\Omega=S L_{3}(\mathbb{R}) / S L_{3}(\mathbb{Z})$. But $H_{B}=g^{-1} H_{S} g$, therefore the orbit $H_{B} \mathbb{Z}^{3}$ is relatively compact if and only if so is the orbit $H_{S} g \mathbb{Z}^{3}$.

Corollary 2.8. Let $B$ be a real nondegenerate indefinite quadratic form in 3 variables. If (2.6) holds, then $B$ is proportional to a rational form.

Proof. The previous corollary implies that $H_{B} \mathbb{Z}^{3}$ is compact; but since this orbit can be identified with $H_{B} / H_{B} \cap S L_{3}(\mathbb{Z})$, this shows that $H_{B} \cap S L_{3}(\mathbb{Z})$ is Zariski dense in $H_{B}$, which is equivalent to $H_{B}$ being defined over $\mathbb{Q}$, hence the claim.

To derive Theorem 1.1 from the above corollary, one can then observe that if $B$ is a real irrational nondegenerate indefinite quadratic form in $k$ variables and $l<k$ then $\mathbb{R}^{k}$ contains a rational subspace $L$ of dimension $l$ such that the restriction of $B$ 
to $L$ is irrational nondegenerate and indefinite (the proof can be found in [DM1]). Hence the validity of Theorem 1.1 in the case $k=3$ implies the general case.

It is worthwhile to mention that in the paper [Op2] Oppenheim modified his conjecture replacing the claim $\inf _{\mathbf{x} \in \mathbb{Z}^{k} \backslash\{0\}}|B(\mathbf{x})|=0$ with "0 is a non-isolated accumulation point of $B\left(\mathbb{Z}^{k}\right)$ ", which he showed to be equivalent to the density of $B\left(\mathbb{Z}^{k}\right)$ in $\mathbb{R}$. This stronger form of the conjecture was also proved by Margulis [Ma4]. It is not difficult to derive it from Theorem 2.5, first reducing to the case $k=3$ : if $B$ is not proportional to a rational form, the orbit $H_{B} \mathbb{Z}^{3}$ is not closed, hence (by non-existence of intermediate subgroups between $H_{B}$ and $S L_{3}(\mathbb{R})$ ) it is dense in $\Omega$, and the density of $B\left(\mathbb{Z}^{3}\right)$ in $\mathbb{R}$ follows.

Finally let us briefly mention quantitative extensions of the above results. For $B$ as above, an open interval $I \subset \mathbb{R}$ and a positive $T$ one defines

$$
V_{I, B}(T) \stackrel{\text { def }}{=}\left\{\mathbf{x} \in \mathbb{R}^{k} \mid B(\mathbf{x}) \in I,\|\mathbf{x}\| \leq T\right\}
$$

and

$$
N_{I, B}(T) \stackrel{\text { def }}{=} \#\left(\mathbb{Z}^{k} \cap V_{I, B}(T)\right),
$$

then one has $N_{I, B}(T) \rightarrow \infty$ as $T \rightarrow \infty$ for every nonempty $I \subset \mathbb{R}$. The next theorem, a compilation of results from [DM2] and [EMM], describes the growth of this counting function comparing it to the volume of $V_{I, B}(T)$.

Theorem 2.9. Let $B$ be a real nondegenerate indefinite quadratic form of signature $(m, n), m+n>2, m \leq n$, which is not proportional to a rational form, and let $I \subset \mathbb{R}$ be a nonempty open interval. Then

(a) [DM2] $\liminf _{T \rightarrow \infty} \frac{N_{I, B}(T)}{\left|V_{I, B}(T)\right|}=1$;

(b) $[\mathrm{EMM}]$ if $n \geq 3$, then $\lim _{T \rightarrow \infty} \frac{N_{I, B}(T)}{\left|V_{I, B}(T)\right|}=1$.

In the exceptional cases, i.e. for forms of signature $(2,1)$ and $(2,2)$, there are counterexamples showing that $N_{I, B}(T)$ can grow like const. $\left|V_{I, B}(T)\right|(\log T)^{1-\varepsilon}$, and it is proved in $[\mathrm{EMM}]$ that const. $\left|V_{I, B}(T)\right| \log T$ is an asymptotically exact upper bound for $N_{I, B}(T)$.

For both parts the crucial step is to approximate the counting function $N_{I, B}(T)$ by values of integrals of certain functions along orbits in the space of lattices. Part (a) relies upon Ratner's uniform distribution theorem (a refinement of Theorem 2.5 for one-parameter unipotent subgroups), while the second part involves delicate estimates based on combinatorics of lattices. See [DM1, DM2, EMM, Ma7] for more details on the proofs and further refinements and generalizations.

\section{Metric linear Diophantine approximation and lattices}

The basic object to study in this section will be the set $M_{m \times n}(\mathbb{R})$ of $m \times n$ real matrices. The word "metric" refers to considering solution sets of Diophantine inequalities in terms of the Lebesgue measure, or, when the sets are of measure zero, for finer analysis, in terms of the Hausdorff dimension. We refer the reader to the books [C, H, S3, Sp3] for a detailed exposition.

In what follows, $\psi(\cdot)$ will be a positive non-increasing function $\mathbb{R}_{+} \mapsto \mathbb{R}_{+}$. We are going to use it to measure the precision of approximation of a real number $\alpha$ 
by rational numbers as follows: we would like the fractional part of $\alpha q$ to be not bigger than $\psi(|q|)$ for infinitely many $q \in \mathbb{Z}$ in order to call $\alpha$ "sufficiently well approximable" (this notion being dependent on $\psi$ ). More precisely, let us say that $\alpha$ is $\psi$-approximable if there are infinitely many $q \in \mathbb{Z}$ such that

$$
|\alpha q+p| \leq \psi(|q|) \quad \text { for some } p \in \mathbb{Z} \text {. }
$$

In order to consider a matrix analogue of this notion, one needs to choose a norm on $\mathbb{R}^{k}$ (we will do it by setting $\|\mathbf{x}\|=\max _{1 \leq i \leq k}\left|x_{i}\right|$ ). Then one says that a matrix $Y \in M_{m \times n}(\mathbb{R})$ (viewed as a system of $m$ linear forms in $n$ variables) is $\psi$-approximable if there are infinitely many $\mathbf{q} \in \mathbb{Z}^{n}$ such that

$$
\|Y \mathbf{q}+\mathbf{p}\|^{m} \leq \psi\left(\|\mathbf{q}\|^{n}\right) \text { for some } \mathbf{p} \in \mathbb{Z}^{m} .
$$

The above normalization (raising norms in the power equal to the dimension of the space, instead of the traditional $\|Y \mathbf{q}+\mathbf{p}\| \leq \psi(\|\mathbf{q}\|)$ as in [Dod] or [BD]) is convenient for many reasons: in our opinion it makes the structure more transparent and less dimension-dependent, and simplifies the connection with homogeneous flows.

The whole theory starts from a positive result of Dirichlet, namely

Theorem 3.1. Every $Y \in M_{m \times n}(\mathbb{R})$ is $\psi_{0}$-approximable, where $\psi_{0}(x)=\frac{1}{x}$.

Clearly the faster $\psi$ decays, the smaller is the set of $\psi$-approximable matrices. The next theorem, Groshev's [Gr] generalization of earlier results of Khintchine, provides the zero-one law for the Lebesgue measure of this set:

Theorem 3.2 (The Khintchine-Groshev Theorem). Almost every (resp. almost no) $Y \in M_{m \times n}(\mathbb{R})$ is $\psi$-approximable, provided the sum $\sum_{l=1}^{\infty} \psi(l)$ diverges (resp. converges).

Now say that $Y \in M_{m \times n}(\mathbb{R})$ is badly approximable if it is not $c \psi_{0}$-approximable for some $c>0$; that is, if there exists $c>0$ such that $\|Y \mathbf{q}+\mathbf{p}\|^{m}\|\mathbf{q}\|^{n} \geq c$ for all $\mathbf{p} \in \mathbb{Z}^{m}$ and all but finitely many $\mathbf{q} \in \mathbb{Z}^{n}$ (equivalently: all $\mathbf{q} \in \mathbb{Z}^{n} \backslash\{0\}$ ).

Note that in the case $m=n=1, \alpha \in \mathbb{R}$ is badly approximable if and only if coefficients in the continued fraction expansion of $\alpha$ are bounded. Using continued fractions, Jarnik proved in 1928 that badly approximable numbers form a set of Hausdorff dimension one; for arbitrary $m, n$ the corresponding fact, i.e. full Hausdorff dimension of badly approximable systems, was established by Schmidt in 1969 [S2].

The following interpretation of this property in terms of homogeneous dynamics is due to Dani. Throughout this section we will fix $m, n \in \mathbb{N}$ and put $k=m+n$.

Theorem 3.3 [D1]. $Y \in M_{m \times n}(\mathbb{R})$ is badly approximable iff the trajectory $\left\{g_{t} L_{Y} \mathbb{Z}^{k} \mid t \in \mathbb{R}_{+}\right\}$, with $L_{Y}$ as in (1.3) and $g_{t}$ as in (2.1), is bounded in the space $\Omega$ of unimodular lattices in $\mathbb{R}^{k}$.

Instead of giving the proof (which, besides the original paper [D1] can be found in $[\mathrm{K} 2, \mathrm{~K} 3]$ ) let us point out the similarity between the above theorem and Lemma 2.7 . Indeed, denote by $S$ the function on $\mathbb{R}^{k}$ given by

$$
S\left(x_{1}, \ldots, x_{k}\right)=\max \left(\left|x_{1}\right|, \ldots,\left|x_{m}\right|\right)^{m} \max \left(\left|x_{m+1}\right|, \ldots,\left|x_{k}\right|\right)^{n} .
$$


Then $Y$ is badly approximable iff for some $c>0$ one has

$$
S\left(L_{Y} \mathbf{x}\right) \geq c \text { for all } \mathbf{x}=(\mathbf{p}, \mathbf{q}) \in \mathbb{Z}^{m} \times\left(\mathbb{Z}^{n} \backslash\{0\}\right) .
$$

Furthermore, the one-parameter group $\left\{g_{t}\right\}$ as in (2.1) is essentially (up to the compact part) the stabilizer of $S$, and, as in the proof of Lemma 2.7, one can show that (3.2) is equivalent to the norm of $g_{t} L_{Y} \mathbf{x}$ being bounded away from zero for all $\mathbf{x} \in \mathbb{Z}^{k} \backslash\{0\}$ and $t \geq 0$, that is, to the statement that $\left\{g_{t} L_{Y} \mathbb{Z}^{k} \mid t \in \mathbb{R}_{+}\right\} \cap \Omega_{\varepsilon}=\varnothing$ for some $\varepsilon>0$.

From the above theorem and the aforementioned result of Schmidt, Dani derived

Corollary 3.4. The set

$$
\left\{\Lambda \in \Omega \mid\left\{g_{t} \Lambda \mid t \geq 0\right\} \text { is bounded }\right\},
$$

with $\left\{g_{t}\right\}$ as in (2.1), has full Hausdorff dimension.

Proof. Indeed, any $\Lambda \in \Omega$ can be written as $\left(\begin{array}{cc}B & 0 \\ C & D\end{array}\right) L_{Y} \mathbb{Z}^{k}$, therefore one has

$$
g_{t} \Lambda=g_{t}\left(\begin{array}{cc}
B & 0 \\
C & D
\end{array}\right) g_{-t} \cdot g_{t} L_{Y} \mathbb{Z}^{k}
$$

But as we saw in one of the examples of $\S 2,\left\{L_{Y} \mid Y \in M_{m \times n}(\mathbb{R})\right\}$ is the expanding horospherical subgroup of $G$ relative to $g_{1}$; thus the conjugation of the neutral and contracting parts plays no role and the trajectory $g_{t} \Lambda$ is bounded iff so is $g_{t} L_{Y} \mathbb{Z}^{k}$.

A possibility to generalize the statement of the last corollary (to actions of other one-parameter groups on other homogeneous spaces) was mentioned by Dani in [D2] and later conjectured by Margulis [Ma5, Conjecture (A)]. The latter conjecture was settled by Margulis and the author in 1996. Let us state here the following weakened version:

Theorem 3.5 [KM1, K4]. Let $G$ be a Lie group, $\Gamma$ a lattice in $G, F=\left\{g_{t} \mid t \geq 0\right\}$ be a one-parameter subsemigroup of $G$ consisting of semisimple ${ }^{1}$ elements, and let $H=H_{+}\left(g_{1}\right)$ be the expanding horospherical subgroup corresponding to $F$. Assume in addition that the $F$-action on $G / \Gamma$ is mixing. Then for any closed $F$-invariant null subset $Z$ of $G / \Gamma$ and any $x \in G / \Gamma$, the set

$$
\{h \in H \mid F h x \text { is bounded and } \overline{F h x} \cap Z=\varnothing\}
$$

has full Hausdorff dimension. In particular, if $\left\{g_{t}\right\}$ is partially hyperbolic, then the set $\{x \in G / \Gamma \mid F x$ is bounded and $\overline{F x} \cap Z=\varnothing\}$ has full Hausdorff dimension.

Note that abundance of exceptional orbits is a feature of many chaotic dynamical systems. See e.g. [AN1, AN2, D4, Dol1, U]. In the situation of Theorem 3.5, the construction of bounded orbits (or, more generally, orbits staying away from a fixed part of the space) comes from uniform distribution of images of expanding leaves (Theorem 2.3). More precisely, first one reduces the problem to the case (2.4), and

${ }^{1} g \in G$ is called semisimple if the operator $\operatorname{Ad}_{g}$ is diagonalizable over $\mathbb{C}$ 
then considers natural "rectangular" partitions of $H$ (called tessellations in [KM1] and [K1]) and studies their behavior under the automorphism $h \mapsto g_{t} h g_{-t}$ of $H$. Theorem 2.3 is used to show than one can cover the set of "bad" points by relatively small number of rectangles. Then those rectangles are used to create a Cantor set consisting of points with orbits avoiding $Z$ and staying within a compact subset of $G / \Gamma$. See $[\mathrm{KM} 1, \mathrm{~K} 1, \mathrm{~K} 4]$ for details and generalizations ${ }^{2}$

So far we have illustrated the impact of ideas coming from Diophantine approximation to ergodic theory. On the other hand, Theorem 3.5 and Dani's correspondence (Theorem 3.3) can be used as an alternative proof of the aforementioned result of Schmidt on abundance of badly approximable systems of linear forms. What follows is another application to number theory, which produces a new result and demonstrates the power of ideas relating the two fields.

Let us consider an inhomogeneous twist of approximation of real numbers by rationals. Instead of just one real number $\alpha$ take a pair $\langle\alpha, \beta\rangle$, consider an affine form $x \mapsto \alpha x+\beta$ and look at fractional parts of its values at integers. Similarly, a system of $m$ affine forms in $n$ variables will be then given by a pair $\langle Y, \mathbf{b}\rangle$, where $Y \in M_{m \times n}(\mathbb{R})$ and $\mathbf{b} \in \mathbb{R}^{m}$. Let us denote by $\tilde{M}_{m \times n}(\mathbb{R})$ the direct product of $M_{m \times n}(\mathbb{R})$ and $\mathbb{R}^{m}$. Now say that a system of affine forms given by $\langle Y, \mathbf{b}\rangle \in$ $\tilde{M}_{m \times n}(\mathbb{R})$ is $\psi$-approximable if there are infinitely many $\mathbf{q} \in \mathbb{Z}^{n}$ such that

$$
\|Y \mathbf{q}+\mathbf{b}+\mathbf{p}\|^{m} \leq \psi\left(\|\mathbf{q}\|^{n}\right) \quad \text { for some } \mathbf{p} \in \mathbb{Z}^{m},
$$

and badly approximable if it is not $c \psi_{0}$-approximable for some $c>0$; that is, there exists a constant $\tilde{c}>0$ such that for every $\mathbf{p} \in \mathbb{Z}^{m}$ and all but finitely many $\mathbf{q} \in \mathbb{Z}^{n} \backslash\{0\}$ one has

$$
\|Y \mathbf{q}+\mathbf{b}+\mathbf{p}\|^{m}\|\mathbf{q}\|^{n}>\tilde{c} .
$$

It can be proved (and follows from an inhomogeneous version of the KhintchineGroshev Theorem, see $[\mathrm{C}])$ that the set of badly approximable $\langle Y, \mathbf{b}\rangle \in \tilde{M}_{m \times n}(\mathbb{R})$ is of measure zero. However, all known examples of badly approximable $\langle Y, \mathbf{b}\rangle \in$ $\tilde{M}_{m \times n}(\mathbb{R})$ belong to a countable union of proper submanifolds of $\tilde{M}_{m \times n}(\mathbb{R})$, hence form a set of positive Hausdorff codimension. Yet a modification of the dynamical approach described above works in this case as well. Namely, one considers a collection of vectors

$$
\left\{\left(\begin{array}{c}
Y \mathbf{q}+\mathbf{b}+\mathbf{p} \\
\mathbf{q}
\end{array}\right) \mid \mathbf{p} \in \mathbb{Z}^{m}, \mathbf{q} \in \mathbb{Z}^{n}\right\}=L_{Y} \mathbb{Z}^{k}+\left(\begin{array}{c}
\mathbf{b} \\
0
\end{array}\right)
$$

which is an element of the space $\hat{\Omega}=\hat{G} / \hat{\Gamma}$ of affine lattices in $\mathbb{R}^{k}$, where

$$
\hat{G} \stackrel{\text { def }}{=} \operatorname{Aff}\left(\mathbb{R}^{k}\right)=G \ltimes \mathbb{R}^{k} \text { and } \hat{\Gamma} \stackrel{\text { def }}{=} \Gamma \ltimes \mathbb{Z}^{k} .
$$

In other words,

$$
\hat{\Omega} \cong\left\{\Lambda+\mathbf{w} \mid \Lambda \in \Omega, \mathbf{w} \in \mathbb{R}^{k}\right\} .
$$

Note that the quotient topology on $\hat{\Omega}$ coincides with the natural topology on the space of affine lattices: that is, $\Lambda_{1}+\mathbf{w}_{1}$ and $\Lambda_{2}+\mathbf{w}_{2}$ are close to each other

\footnotetext{
${ }^{2}$ In particular, it follows from the methods of [KM1] that one can remove the assumption of semisimplicity of elements of $F$, but then one needs the $F$-action to be exponentially mixing, that is, (2.2) must hold for any $\varphi, \psi$ as in Theorem 2.2 .
} 
if so are $\mathbf{w}_{i}$ and the generating elements of $\Lambda_{i}$. Note also that $\hat{\Omega}$ is non-compact and has finite Haar measure, and that $\Omega$ (the set of true lattices) can be identified with a subset of $\hat{\Omega}$ (affine lattices containing the zero vector). Finally, $g_{t}$ as in (2.1) acts on $\hat{\Omega}$, and it is not hard to show that the expanding horospherical subgroup corresponding to $g_{1}$ is exactly the set of all elements of $\hat{G}$ with linear part $L_{Y}$ and translation part $\left(\begin{array}{l}\mathbf{b} \\ 0\end{array}\right), Y \in M_{m \times n}(\mathbb{R})$ and $\mathbf{b} \in \mathbb{R}^{m}$.

Now, for $\varepsilon>0$, define

$$
\hat{\Omega}_{\varepsilon} \stackrel{\text { def }}{=}\{\Lambda \in \hat{\Omega} \mid\|\mathbf{x}\|<\varepsilon \text { for some } \mathbf{x} \in \Lambda\}
$$

Then $\hat{\Omega} \backslash \hat{\Omega}_{\varepsilon}$ is a closed (non-compact) set disjoint from $\Omega$.

Theorem 3.6 [K4]. Let $F=\left\{g_{t} \mid t \geq 0\right\}$ be as in (2.1). Then

$$
\begin{gathered}
F\left(L_{Y} \mathbb{Z}^{k}+\left(\begin{array}{c}
\mathbf{b} \\
0
\end{array}\right)\right) \quad \text { is bounded and stays away from } \Omega \\
\Downarrow \\
F\left(L_{Y} \mathbb{Z}^{k}+\left(\begin{array}{c}
\mathbf{b} \\
0
\end{array}\right)\right) \subset \hat{\Omega} \backslash \hat{\Omega}_{\varepsilon} \text { for some } \varepsilon>0 \\
\Downarrow \\
\langle Y, \mathbf{b}\rangle \text { is badly approximable }
\end{gathered}
$$

The proof is basically a slight modification of ideas involved in the proof of Theorem 3.3. It follows from the results of $[\mathrm{BM}]$ (see also [Ma5]) that the $F$-action on $\hat{\Omega}$ is mixing. Since $\Omega \subset \hat{\Omega}$ is closed, null and $g_{t}$-invariant, Theorem 3.5 applies and one gets

Corollary 3.7. The set of badly approximable $\langle Y, \mathbf{b}\rangle \in \tilde{M}_{m \times n}(\mathbb{R})$ has full Hausdorff dimension.

See $[\mathrm{K} 4]$ for details, remarks and extensions.

We close the section by stating a theorem generalizing Dani's correspondence (Theorem 3.3) to $\psi$-approximable systems. First we need a simple "change of variables" lemma.

Lemma 3.8. Fix $m, n \in \mathbb{N}$ and $x_{0}>0$, and let $\psi:\left[x_{0}, \infty\right) \mapsto(0, \infty)$ be a nonincreasing continuous function. Then there exists a unique continuous function $\varepsilon:\left[t_{0}, \infty\right) \mapsto(0, \infty)$, where $e^{k t_{0}}=x_{0}^{m} / \psi\left(x_{0}\right)^{n}$, such that

$$
\text { the function } t \mapsto e^{t} \varepsilon(t)^{n} \quad \text { is strictly increasing and unbounded, }
$$

$$
\text { the function } \quad t \mapsto e^{-t} \varepsilon(t)^{m} \quad \text { is nonincreasing, }
$$

and

$$
\psi\left(e^{t} \varepsilon(t)^{n}\right)=e^{-t} \varepsilon(t)^{m} \quad \forall t \geq t_{0}
$$


Conversely, given $t_{0} \in \mathbb{R}$ and a continuous function $\varepsilon:\left[t_{0}, \infty\right) \mapsto(0, \infty)$ such that (3.3ab) hold, there exists a unique continuous non-increasing function $\psi:\left[x_{0}, \infty\right) \mapsto$ $(0, \infty)$, with $x_{0}=e^{t_{0}} \varepsilon\left(t_{0}\right)^{n}$, satisfying (3.4).

See [KM3] for the proof. In many cases one can explicitly solve (3.4) to express $\varepsilon(\cdot)$ knowing $\psi(\cdot)$ and vice versa. For example if $\psi(x)=c \psi_{0}(x)=c / x$, the equation (3.4) gives $c e^{-t} \varepsilon(t)^{-n}=e^{-t} \varepsilon(t)^{m}$, and one sees that the corresponding function $\varepsilon$ is constant (more precisely, $\varepsilon(t) \equiv c^{1 / k}$ ). Or one can take $\psi(x)=c \psi_{\beta}(x)$, where $\psi_{\beta}(x)=\frac{1}{x^{1+\beta}}, \beta>0$; then $\varepsilon(t)$ decreases exponentially, namely

$$
\varepsilon(t)=c^{\gamma / \beta} e^{-\gamma t}, \text { where } \gamma=\frac{\beta}{(1+\beta) n+m} .
$$

Now we can state a generalization of Theorem 3.3:

Theorem 3.9. $Y \in M_{m \times n}(\mathbb{R})$ is $\psi$-approximable iff there exist arbitrarily large positive $t$ such that $g_{t} L_{Y} \mathbb{Z}^{k} \in \Omega_{\varepsilon(t)}$, where $\left\{g_{t}\right\}$ is as in (2.1), $L_{Y}$ as in (1.3), and $\varepsilon(\cdot)$ is the function corresponding to $\psi$ as in the previous lemma.

Loosely speaking, good rational approximations for $Y$ correspond to far excursions of the orbit into the "cusp neighborhoods" $\Omega_{\varepsilon}$. In other words, one can measure the "growth rate" of the orbit in terms of hitting the sets $\Omega_{\varepsilon(t)}$ in time $t$ for infinitely many $t \in \mathbb{N}$, and fast-growing orbits would correspond to systems approximable with a fast-decaying approximation function.

It is shown in [KM3] how the above correspondence provides an alternative (dynamical) proof of Theorem 3.2. More precisely, one can use ergodic properties of the $g_{t}$-action on $\Omega$ (exponential decay of correlations, see Theorem 2.2) to prove the following

Theorem 3.10. Let $\varepsilon(\cdot)$ be any positive function. Then for almost all (resp. almost no) $\Lambda \in \Omega$ one has $g_{t} \Lambda \in \Omega_{\varepsilon(t)}$ for infinitely many $t \in \mathbb{N}$, provided the sum

$$
\sum_{t=1}^{\infty} \varepsilon(t)^{k}
$$

diverges (resp. converges).

We remark that the ratio $\mu\left(\Omega_{\varepsilon}\right) / \varepsilon^{k}$ is shown in [KM3] to be bounded from both sides; therefore the sum (3.5) is finite/infinite iff so is $\sum_{t=1}^{\infty} \mu\left(\Omega_{\varepsilon(t)}\right)$. This places the above theorem in the rank of Borel-Cantelli type results. See [KM3] for generalizations and applications, and [CK, CR, Dol2, Ph, Su] for other results of similar flavor.

Another application of the correspondence of Theorem 3.9 will be given in the next section.

\section{Diophantine approximation ON MANifolds}

We start from the setting of the previous section but specialize to the case $m=1$; that is, to Diophantine approximation of just one linear form given by $\mathbf{y} \in \mathbb{R}^{n}$. Recall that Theorem 3.2 says that whenever $\sum_{l=1}^{\infty} \psi(l)$ is finite, almost every $\mathbf{y}$ is not $\psi$-approximable; that is, the inequality

$$
|\mathbf{q} \cdot \mathbf{y}+p| \leq \psi\left(\|\mathbf{q}\|^{n}\right)
$$


has at most finitely many solutions. It is instructive to sketch an elementary proof: for fixed $p$, q, the set of $\mathbf{y}$ satisfying (4.1) is a $\frac{\psi\left(\|\mathbf{q}\|^{n}\right)}{\|\mathbf{q}\|}$-neighborhood of a hyperplane

$$
\mathbf{q} \cdot \mathbf{y}+p=0
$$

thus if one restricts $\mathbf{y}$ to lie in $[0,1]^{n}$ (or any other bounded subset of $\mathbb{R}^{n}$ ), the set of solutions will have measure at most const. $\frac{\psi\left(\|\mathbf{q}\|^{n}\right)}{\|\mathbf{q}\|}$. Since there are at most const. $\|\mathbf{q}\|$ admissible values of $p$, the sum of measures of all sets of solutions is at most

$$
\sum_{\mathbf{q} \in \mathbb{Z}^{n}} \psi\left(\|\mathbf{q}\|^{n}\right) \asymp \sum_{l=1}^{\infty} l^{n-1} \psi\left(l^{n}\right) \asymp \sum_{l=1}^{\infty} \psi(l),
$$

and the proof is finished by an application of the Borel-Cantelli Lemma.

Recall that $\psi_{\beta}(x)=x^{-(1+\beta)}, \beta>0$, was one of the examples of functions realizing the convergence case in the Khintchine-Groshev Theorem. Say that $\mathbf{y} \in$ $\mathbb{R}^{n}$ is very well approximable (VWA) if it is $\psi_{\beta}$-approximable for some $\beta>0$. Thus almost all $\mathbf{y} \in \mathbb{R}^{n}$ are not VWA.

Now consider the following problem, raised by Mahler in 1932 [M]: is it true that for almost all $x \in \mathbb{R}$ the inequality

$$
\left|p+q_{1} x+q_{2} x^{2}+\cdot+q_{n} x^{n}\right| \leq\|\mathbf{q}\|^{-n(1+\beta)}
$$

has at most finitely many solutions? In other words, for a.e. $x \in \mathbb{R}$, the $n$-tuple

$$
\mathbf{y}(x)=\left(x, x^{2}, \ldots, x^{n}\right)
$$

is not VWA. The proof presented above does not work, since this time one has to estimate the measure of intersection of the curve (4.3) with the sets of solutions of inequalities (4.1), and for some choices of $p, \mathbf{q}$ (namely for those which make the hyperplane (4.2) nearly tangent to the curve (4.3)) it is hard to produce a reasonable estimate.

This problem stood open for more than 30 years until it was solved in 1964 by Sprindžuk [Sp1, Sp2]. Earlier several special cases were considered, and, quoting Sprindžuk's survey paper, the problem rapidly revealed itself to be non-trivial and involving "deep and complicated phenomena in which arithmetical properties of numbers are closely entangled with combinatorial-topological properties" of the curve. The solution to Mahler's problem has eventually led to the development of a new branch of metric number theory, usually referred to as "Diophantine approximation with dependent quantities" or "Diophantine approximation on manifolds". We invite the reader to look at Sprindžuk's monographs [Sp2, Sp3] and a recent book [BD] for a systematic exposition of the field.

Mahler's problem and its generalizations have several motivations. The original motivation of Mahler comes from transcendental number theory. Indeed, the $n$ tuple (4.3) is not VWA if and only if for every $\beta>0$ there are at most finitely many polynomials $P \in \mathbb{Z}[x]$ with degree at most $n$ such that $|P(x)|<h(P)^{-n(1+\beta)}$, where $h(P)$ is the height of $P$; loosely speaking, $x$ is "not very algebraic", and the affirmative solution to the problem shows that almost all $x$ are such.

Another motivation comes from KAM theory: it is known that behavior of perturbation of solutions of ODEs is related to Diophantine properties of coefficients. 
If the latter are restricted to lie on a curve or submanifold of $\mathbb{R}^{n}$, it may be important to know that almost all values have certain approximation properties. See [de la Llave's lectures, this volume] and [BD, Chapter 7].

However, from the author's personal viewpoint, the appeal of this branch of number theory lies in its existing and potential generalizations. In a sense, the affirmative solution to Mahler's problem shows that a certain property of $\mathbf{y} \in \mathbb{R}^{n}$ (being not VWA) which holds for generic $\mathbf{y} \in \mathbb{R}^{n}$ in fact holds for generic points on the curve (4.3). In other words, the curve inherits the above Diophantine property from the ambient space, unlike, for example, a line $\mathbf{y}(x)=(x, \ldots, x)$ - it is clear that every point on this line is VWA. This gives rise to studying other subsets of $\mathbb{R}^{n}$ and other Diophantine properties, and looking at whether this inheritance phenomenon takes place.

Note that the curve (4.3) is not contained in any affine subspace of $\mathbb{R}^{n}$ (in other words, constitutes an essentially $n$-dimensional object). The latter property, or, more precisely, its infinitesimal analogue, is formalized in the following way. Let $V$ be an open subset of $\mathbb{R}^{d}$. Say that an $n$-tuple $\mathbf{f}=\left(f_{1}, \ldots, f_{n}\right)$ of $C^{l}$ functions $V \mapsto \mathbb{R}$ is nondegenerate at $x \in V$ if the space $\mathbb{R}^{n}$ is spanned by partial derivatives of $\mathbf{f}$ at $x$ of order up to $l$. If $M \subset \mathbb{R}^{n}$ is a $d$-dimensional smooth submanifold, we will say that $M$ is nondegenerate at $\mathbf{y} \in M$ if any (equivalently, some) diffeomorphism f between an open subset $V$ of $\mathbb{R}^{d}$ and a neighborhood of $\mathbf{y}$ in $M$ is nondegenerate at $\mathbf{f}^{-1}(\mathbf{y})$. We will say that $\mathbf{f}: V \rightarrow \mathbb{R}^{n}$ (resp. $M \subset \mathbb{R}^{n}$ ) is nondegenerate if it is nondegenerate at almost every point of $V$ (resp. $M$, in the sense of the natural measure class on $M$ ). If the functions $f_{i}$ are analytic, it is easy to see that the linear independence of $1, f_{1}, \ldots, f_{n}$ over $\mathbb{R}$ in $V$ is equivalent to all points of $M=\mathbf{f}(U)$ being nondegenerate. Thus the above nondegeneracy condition can be viewed as an infinitesimal version of not lying in any proper affine subspace of $\mathbb{R}^{n}$.

It appears that many known and anticipated results in the field fall in the framework of the following vague

Meta-Conjecture. "Any" Diophantine property of vectors in an ambient space (e.g. $\mathbb{R}^{n}$ ) which holds for almost all points in this space should hold for generic points on a nondegenerate smooth submanifold $M$ of the space.

It was conjectured in 1980 by Sprindžuk [Sp4, Conjecture $\mathrm{H}_{1}$ ] that almost all points on a nondegenerate analytic submanifold of $\mathbb{R}^{n}$ are not VWA. This conjecture was supported before and after 1980 by a number of partial results, one of the first being Schmidt's proof [S1] for nondegenerate planar curves. The general case was settled in 1998 by Margulis and the author using the dynamical approach. Namely, the following was proved:

Theorem 4.1 [KM2]. Let $M$ be a nondegenerate smooth submanifold of $\mathbb{R}^{n}$. Then almost all points of $M$ are not VWA.

This is the result we will focus on later in this section.

In another direction, Sprindžuk's solution to Mahler's problem was improved in 1964 by Baker [B1] and later (1984) by Bernik [Bern, BD]; the latter proved that whenever $\sum_{l=1}^{\infty} \psi(l)$ is finite, almost all points of the curve (4.3) are not $\psi$ approximable. And several years ago Beresnevich [Bere1] proved the divergence counterpart, thus establishing a complete analogue of the Khintchine-Groshev Theorem for the curve (4.3). 
It turned out that a modification of the methods from [KM2] allows one to prove the convergence part of the Khintchine-Groshev Theorem for any nondegenerate manifold. In other words, the following is true:

Theorem 4.2. Let $M$ be a nondegenerate smooth submanifold of $\mathbb{R}^{n}$ and let $\psi$ be such that $\sum_{l=1}^{\infty} \psi(l)$ is finite. Then almost all points of $M$ are not $\psi$-approximable.

This is proved in [BKM] and also independently in [Bere2]. A work on the divergence case is currently in progress. (We note that the main result of [BKM] has a stronger "multiplicative" version (see Theorem 5.3) which is currently not doable by classical Sprindžuk-style methods developed in [Bere2].)

In this survey we will indicate a proof of Theorem 4.1 by first restating it in the language of flows on the space of lattices. For this we set $k=n+1$ and look at the one-parameter group

$$
g_{t}=\operatorname{diag}\left(e^{t}, e^{-t / n}, \ldots, e^{-t / n}\right)
$$

acting on $\Omega=S L_{k}(\mathbb{R}) / S L_{k}(\mathbb{Z})$, and given $\mathbf{y} \in \mathbb{R}^{n}$, consider $L_{\mathbf{y}} \stackrel{\text { def }}{=}\left(\begin{array}{ll}1 & \mathbf{y}^{T} \\ 0 & I_{n}\end{array}\right)$ (cf. (2.1) and (1.3)). It follows from Theorem 3.9 and the example discussed afterwards that $\mathbf{y} \in \mathbb{R}^{n}$ is VWA iff for some $\gamma>0$ there exist arbitrarily large positive $t$ such that

$$
g_{t} L_{\mathbf{y}} \mathbb{Z}^{k} \in \Omega_{e^{-\gamma t}} .
$$

Equivalently, for some $\gamma>0$ there are infinitely many $t \in \mathbb{N}$ such that (4.5) holds.

With this in mind, let us turn to the setting of Theorem 4.1. Namely let $V$ be an open subset of $\mathbb{R}^{d}$ and $\mathbf{f}=\left(f_{1}, \ldots, f_{n}\right)$ an $n$-tuple of $C^{k}$ functions $V \mapsto \mathbb{R}$ which is nondegenerate at almost every point of $V$. The theorem would be proved if we show that for any $\gamma>0$ the set

$$
\left\{x \in V \mid g_{t} L_{\mathbf{f}(x)} \mathbb{Z}^{k} \in \Omega_{e^{-\gamma t}} \text { for infinitely many } t \in \mathbb{N}\right\}
$$

has measure zero. In other words, a submanifold $\mathbf{f}(V)$ of $\mathbb{R}^{n}$ gives rise to a submanifold $L_{\mathbf{f}(V)} \mathbb{Z}^{k}$ of the space of lattices, and one needs to show that the growth rate of generic orbits originating from this submanifold is consistent with the growth rate of an orbit of a generic point of $\Omega$ (see Theorem 3.10 for an explanation of why lattices $\Lambda$ such that $g_{t} \Lambda \in \Omega_{e^{-\gamma t}}$ for infinitely many $t \in \mathbb{N}$ form a null subset of $\Omega$ ).

Now one can use the Borel-Cantelli Lemma to reduce Theorem 4.1 to the following statement:

Theorem 4.3. Let $V$ be an open subset of $\mathbb{R}^{d}$ and $\mathbf{f}=\left(f_{1}, \ldots, f_{n}\right)$ an $n$-tuple of $C^{k}$ functions $V \mapsto \mathbb{R}$ which is nondegenerate at $x_{0} \subset V$. Then there exists a neighborhood $B$ of $x_{0}$ contained in $V$ such that for any $\gamma>0$ one has

$$
\sum_{t=1}^{\infty}\left|\left\{x \in B \mid g_{t} L_{\mathbf{f}(x)} \mathbb{Z}^{k} \in \Omega_{e^{-\gamma t}}\right\}\right|<\infty .
$$

Here is the turning point of the argument: $t$-dynamics gives way to $x$-dynamics, namely, a natural way to demonstrate (4.6) is to fix $t$ and think of the set $\left\{g_{t} L_{\mathbf{f}(x)} \mathbb{Z}^{k}\right\}$ 
as of an orbit of certain action (not a group action!), the goal being to prove that a substantial part of this "orbit" lies outside of "cusp neighborhoods" $\Omega_{e^{-\gamma t}}$ uniformly for all $t$. What immediately comes to mind is the recurrence property of unipotent orbits, that is, Theorem 2.4. And it turns out that a modification of the argument used to prove the latter theorem allows one to estimate the amount of "time" $x$ that the "trajectory" $x \mapsto g_{t} L_{\mathbf{f}(x)} \mathbb{Z}^{k}$ spends "close to infinity" in $\Omega$. More precisely, the following can be proved:

Theorem 4.4. Let $V, \mathbf{f}$ and $x_{0}$ be as in Theorem 4.3. Then there exists a neighborhood $B$ of $x_{0}$ contained in $V$ and constants $C, \rho>0$ such that for any $\varepsilon>0$ and any positive $t$ one has

$$
\left|\left\{x \in B \mid g_{t} L_{\mathbf{f}(x)} \mathbb{Z}^{k} \in \Omega_{\varepsilon}\right\}\right|<C \varepsilon^{1 / d n}|B| .
$$

It is straightforward to verify that Theorem 4.3 follows from the above uniform estimate.

It remains to explain why the behavior of the curve $x \mapsto g_{t} L_{\mathbf{f}(x)} \mathbb{Z}^{k}$ is similar to that of the unipotent orbit. In fact it has been understood a long time ago that the main property of the unipotent actions on which the recurrence estimates are based is the polynomial dependence of $u_{x}$ on $x$ (see [Ma2, Sh]). One may wonder what is so special about polynomials - and it turns out that the crucial property is roughly "not making very sharp turns". More precisely, here is the definition motivated by the analysis of the proofs in [Ma2] (see also [EMS]): for $C, \alpha>0$ say that a continuous function $f$ on an open set $V \subset \mathbb{R}^{d}$ is $(C, \alpha)$-good on $V$ if for any open ball $B \subset V$ and any positive $\varepsilon$ one has

$$
\left|\left\{x \in B|| f(x)\left|<\varepsilon \cdot \sup _{x \in B}\right| f(x) \mid\right\}\right| \leq C \varepsilon^{\alpha}|B| .
$$

In other words, a good function which takes small values on a big part of a ball is not allowed to grow very fast on the remaining part of the ball. The main example is provided by polynomials:

Lemma 4.5. Any polynomial $f \in \mathbb{R}[x]$ of degree not greater than $k$ is $(4 k, 1 / k)$ $\operatorname{good}$ on $\mathbb{R}$.

This easily follows from Lagrange's interpolation formula, see [DM2, KM2]. The next theorem (the main result of [KM2]) therefore provides a generalization of Theorem 2.4 to polynomial trajectories on $\Omega$. To state it we need to introduce some notation. If $\Delta$ is a discrete subgroup of $\mathbb{R}^{k}$ (not necessarily a lattice) generated by $\mathbf{v}_{1}, \ldots, \mathbf{v}_{l}$, let us measure its norm, $\|\Delta\|$, by the norm of the exterior product $\mathbf{v}_{1} \wedge \cdots \wedge \mathbf{v}_{l}$. For this one needs to extend the norm from $\mathbb{R}^{k}$ to its exterior algebra. If $\mathbf{e}_{1}, \ldots, \mathbf{e}_{k}$ are standard base vectors of $\mathbb{R}^{k}$, the elements $\mathbf{e}_{I} \stackrel{\text { def }}{=} \mathbf{e}_{i_{1}} \wedge \cdots \wedge \mathbf{e}_{i_{l}}$, $I=\left\{i_{1}, \ldots, i_{l}\right\} \subset\{1, \ldots, k\}$ form a basis of $\bigwedge^{l}\left(\mathbb{R}^{k}\right)$. Since Diophantine applications call for the supremum norm, we will extend $\|\cdot\|$ to $\bigwedge\left(\mathbb{R}^{k}\right)$ by setting $\left\|\sum_{I} w_{I} \mathbf{e}_{I}\right\|=$ $\max _{I}\left|w_{I}\right|$.

Now let us consider a curve in $\Omega$ given by $x \mapsto h(x) \mathbb{Z}^{k}$, where $h$ is some function from $\mathbb{R}^{d}$ to $G L_{k}(\mathbb{R})$. It turns out that in order to understand its recurrence properties one has to keep an eye on norms of all discrete subgroups of $h(x) \mathbb{Z}^{k}$; in particular, it will be necessary to prove that all those norms (as functions of $x$ ) 
are $(C, \alpha)$-good for some $C, \alpha$. In fact, it will suffice to look at the coordinates of $h(x)\left(\mathbf{v}_{1} \wedge \cdots \wedge \mathbf{v}_{l}\right)$ where $\mathbf{v}_{1}, \ldots, \mathbf{v}_{l}$ form a basis of $\Delta \subset \mathbb{Z}^{k}$; one can easily show that if all components of a vector function are $(C, \alpha)$-good, the norm of this function is also $(C, \alpha)$-good.

Theorem 4.6. Let $d, k \in \mathbb{N}, C, \alpha>0,0<\rho \leq 1 / k$, and let a ball $B=B\left(x_{0}, r_{0}\right) \subset$ $\mathbb{R}^{d}$ and a map $h: \tilde{B} \rightarrow G L_{k}(\mathbb{R})$ be given, where $\tilde{B}$ stands for $B\left(x_{0}, 3^{k} r_{0}\right)$. Assume that for any subgroup $\Delta$ of $\mathbb{Z}^{k}$,

(i) the function $x \mapsto\|h(x) \Delta\|$ is $(C, \alpha)$-good on $\tilde{B}$;

(ii) $\sup _{x \in B}\|h(x) \Delta\| \geq \rho$.

Then for any positive $\varepsilon \leq \rho$ one has

$$
\left|\left\{x \in B \mid h(x) \mathbb{Z}^{k} \in \Omega_{\varepsilon}\right\}\right| \leq \operatorname{const}(d, k) \cdot\left(\frac{\varepsilon}{\rho}\right)^{\alpha}|B| .
$$

Corollary 4.7. For any lattice $\Lambda$ in $\mathbb{R}^{k}$ there exists a constant $\rho=\rho(\Lambda)>0$ such that for any one-parameter unipotent subgroup $\left\{u_{x}\right\}_{x \in \mathbb{R}}$ of $S L_{k}(\mathbb{R})$, for any $T>0$ and any $\varepsilon \leq \rho$, one has

$$
\left|\left\{0<x<T \mid u_{x} \Lambda \in \Omega_{\varepsilon}\right\}\right| \leq \operatorname{const}(k)\left(\frac{\varepsilon}{\rho}\right)^{1 / k^{2}} T .
$$

This is clearly a quantitative strengthening of Theorem 2.4, with an explicit estimate of $\delta$ in terms of $\varepsilon$.

Proof. Write $\Lambda$ in the form $g \mathbb{Z}^{k}$ with $g \in G L_{k}(\mathbb{R})$, and denote by $h$ the function $h(x)=u_{x} g$. For any $\Delta \subset \mathbb{Z}^{k}$ with basis $\mathbf{v}_{1}, \ldots, \mathbf{v}_{j}$, the coordinates of $h(x)\left(\mathbf{v}_{1} \wedge\right.$ $\cdots \wedge \mathbf{v}_{j}$ ) will be polynomials in $x$ of degree not exceeding $k^{2}$. Hence the functions $x \mapsto\|h(x) \Delta\|$ will be $\left(C, 1 / k^{2}\right)$-good on $\mathbb{R}$, where $C$ is a constant depending only on $k$. Now let $\rho \stackrel{\text { def }}{=} \min \left(1 / k, \inf _{\Delta \in \mathcal{L}\left(\mathbb{Z}^{k}\right)}\|g \Delta\|\right)$, positive by the discreteness of $\Lambda$ in $\mathbb{R}^{k}$. Then $\|h(0) \Delta\| \geq \rho$ for any $\Delta \subset \mathbb{Z}^{k}$, therefore, with the the substitutions $B=(0, T), \alpha=1 / k^{2}$ and $d=1$ assumptions (i) and (ii) of Theorem 4.6 are satisfied, and one immediately gets (4.9) from (4.8).

As was mentioned in $\S 2$, the proof of Theorem 4.6 is based on delicate combinatorial (partially ordered) structure of the space of lattices, and the reader is referred to [KM2] or [BKM] (most of the ideas are borrowed from [Ma2] and [D3]). Assuming the latter theorem, we conclude by presenting a

Sketch of proof of Theorem 4.4. Take a positive $t$ and consider $h(x) \stackrel{\text { def }}{=} g_{t} L_{\mathbf{f}(x)}$; clearly all one needs to prove (4.7) is to check conditions (i) and (ii) of Theorem 4.6 for every $\Delta \subset \mathbb{Z}^{k}$. An elementary computation shows that the coordinates of $h(x)\left(\mathbf{v}_{1} \wedge \cdots \wedge \mathbf{v}_{l}\right)$ for any choice of vectors $\mathbf{v}_{i}$ are linear combinations of functions $f_{1}, \ldots, f_{n}$ and 1 . Consider first the case of original Mahler's conjecture, with $d=1$ and $f_{i}(x)=x^{i}$. Then, as in the proof of Corollary 4.7, condition (i) is automatic due to Lemma 4.5. Further, a straightforward computation of the action of $h(x)$ on exterior products of vectors in $\mathbb{R}^{k}$ shows that at least one coefficient of at least one polynomial arising as a coordinate must have absolute value not less than 1 . 
This implies that for every interval $B$ there exists a constant $\rho$ (independent of $t$ ) such that (ii) holds.

It remains to pass from this special case to the general situation of functions $f_{1}, \ldots, f_{n}$ on $\mathbb{R}^{d}$ coordinatizing a nondegenerate submanifold of $\mathbb{R}^{n}$. Here one basically has to show that locally these functions behave like polynomials. Indeed, the following was proved in [KM2]:

Lemma 4.8. Let $\mathbf{f}=\left(f_{1}, \ldots, f_{n}\right)$ be a $C^{l}$ map from an open subset $V$ of $\mathbb{R}^{d}$ to $\mathbb{R}^{n}$, and let $x_{0} \in V$ be such that $\mathbb{R}^{n}$ is spanned by partial derivatives of $\mathbf{f}$ at $x_{0}$ of order up to $l$. Then there exists a neighborhood $U \subset V$ of $x_{0}$ and positive $C$ such that any linear combination of $1, f_{1}, \ldots, f_{n}$ is $(C, 1 / d l)$-good on $V$.

Now to finish the proof one simply has to choose $U$ according to the above lemma, then pick a ball $\tilde{B}$ centered at $x_{0}$ and contained in $U$, and finally take $B$ to be a concentric ball with radius $3^{k}$ times smaller. This implies condition (i) with $\alpha=1 / d n$ and some constant $C$ independent of $\Delta$ and $t$, and (ii) follows as a result of a computation described above: one shows that at least one coordinate of $h(x)\left(\mathbf{v}_{1} \wedge \cdots \wedge \mathbf{v}_{l}\right)$ must have the form $c_{0}+\sum_{i=1}^{n} c_{i} f_{i}(x)$ with $\max \left(\left|c_{1}\right|, \ldots,\left|c_{n}\right|\right) \geq 1$, and therefore one gets a lower bound (again independent of $t$ and $\Delta$ ) for $\sup _{x \in B}\|h(x) \Delta\|$.

\section{Multiplicative approximation}

We have already seen in Conjecture 1.2 how the magnitude of the integer vector $\mathbf{q}$ was measured by taking the product of coordinates rather than the maximal coordinate (that is the norm of the vector). Let us formalize it by saying, for $\psi$ as before, that $Y \in M_{m \times n}(\mathbb{R})$ is $\psi$-multiplicatively approximable ( $\psi$-MA) if there are infinitely many $\mathbf{q} \in \mathbb{Z}^{n}$ such that

$$
\Pi(Y \mathbf{q}+\mathbf{p}) \leq \psi\left(\Pi_{+}(\mathbf{q})\right) \quad \text { for some } \mathbf{p} \in \mathbb{Z}^{m} .
$$

where for $\mathbf{x}=\left(x_{1}, \ldots, x_{k}\right) \in \mathbb{R}^{k}$ one defines $\Pi(\mathbf{x})=\prod_{i=1}^{k}\left|x_{i}\right|$ and $\Pi_{+}(\mathbf{x})=$ $\prod_{i=1}^{k} \max \left(\left|x_{i}\right|, 1\right)$. Clearly any $\psi$-approximable system of linear forms is automatically $\psi$-MA, but the converse is not necessarily true. Similarly to the standard setting, one can define badly multiplicatively approximable (BMA) and very well multiplicatively approximable (VWMA) systems. It can be easily shown that almost no $Y \in M_{m \times n}(\mathbb{R})$ are $\psi$-MA if the sum

$$
\sum_{l=1}^{\infty}(\log l)^{k-2} \psi(l)
$$

converges (here we again set $k=m+n$ ); in particular, VWMA systems form a set of measure zero. The converse (i.e. a multiplicative analogue of Theorem 1.2) can be proved using methods of Schmidt; the case $n=1$ is contained in [G].

On the other hand, saying that a vector $\mathbf{y} \in \mathbb{R}^{n}$ (viewed as a linear form $\mathbf{q} \mapsto \mathbf{y} \cdot \mathbf{q}$ ) is not BMA is equivalent to (1.1); in other words, Conjecture 1.2 states that no $\mathbf{y} \in \mathbb{R}^{n}, n \geq 2$, is badly multiplicatively approximable. A more general statement that no $Y \in M_{m \times n}(\mathbb{R})$ is BMA unless $m=n=1$ in fact reduces to this conjecture; moreover, as it is the case with Theorem 1.1, it is enough to prove Conjecture 1.2 for $n=2$.

It seems natural to bring lattices into the game. In fact, one can observe the similarity between the statements of the Oppenheim and Littlewoods's conjectures. 
Indeed, to say that $Y \in M_{m \times n}(\mathbb{R})$ is not BMA amounts to saying that 0 is the infimum of absolute values of a certain homogeneous polynomial at integer points. Similarly to what was done for quadratic forms, a linear change of variables transforms this polynomial into the product of coordinates $\Pi(\mathbf{x}), \mathbf{x} \in \mathbb{R}^{k}$, and according to the scheme developed in the preceding sections, the dynamical system reflecting Diophantine properties of $A$ must come from the group stabilizing $\Pi$, that is, the full diagonal subgroup $D$ of $S L_{k}(\mathbb{R})$. Thus the problems rooted in multiplicative Diophantine approximation bring us to higher rank actions on $\Omega$ (in implicit form this was already noticed in the paper [CS] of Cassels and Swinnerton-Dyer). More precisely, one can state a multiplicative version of generalized Dani's correspondence (Theorem 3.8), relating multiplicative Diophantine properties of $A$ to orbits of the form $\left\{g L_{Y} \mathbb{Z}^{k} \mid g \in D_{+}\right\}$where $D_{+}$is a certain open chamber in the group $D$. (For a version of such a correspondence see [KM3, Theorem 9.2].)

We illustrate this principle by two examples below, where for the sake of simplicity of exposition we specialize to the case $m=1$ (one linear form $\mathbf{q} \mapsto \mathbf{y} \cdot \mathbf{q}, \mathbf{y} \in \mathbb{R}^{n}$ ), setting $k=n+1$. We will need the following notation: for $\mathbf{t}=\left(t_{1}, \ldots, t_{n}\right) \in \mathbb{R}^{n}$ let us denote $\sum_{i=1}^{n} t_{i}$ by $t$ and define

$$
g_{\mathbf{t}}=\operatorname{diag}\left(e^{t}, e^{-t_{1}}, \ldots, e^{-t_{n}}\right) \in S L_{k}(\mathbb{R})
$$

Littlewoods's conjecture. One can show that $\mathbf{y} \in \mathbb{R}^{n}$ satisfies (1.1) (that is, it is BMA) iff the trajectory $\left\{g_{\mathbf{t}} L_{\mathbf{y}} \mathbb{Z}^{k} \mid \mathbf{t} \in \mathbb{R}_{+}^{n}\right\}$ is bounded in the space of lattices in $\mathbb{R}^{k}$. Thus Conjecture 1.2 is equivalent to the statement that every trajectory as above is unbounded. In fact, an argument rooted in the "Isolation Theorem" of Cassels and Swinnerton-Dyer [CS, LW, Ma4] shows that the latter statement can be reduced to the following

Conjecture 5.1. Let $D$ be the subgroup of diagonal matrices in $S L_{k}(\mathbb{R}), k \geq 3$. Then any relatively compact orbit $D \Lambda, \Lambda$ a lattice in $\mathbb{R}^{k}$, is compact.

Notice that Theorem 3.5 shows that the above statement does not hold if $k=2$. This highlights the difference between rank-one and higher rank dynamics. Note also the similarity between Corollary 2.6 and Conjecture 5.1, showing that higher rank hyperbolic actions share some features with unipotent dynamics. In fact, Conjecture 5.1 is a special case of a more general hypothesis, see [Ma8, Conjecture 1], which, roughly speaking, says that for a connected Lie group $G$, a lattice $\Gamma \subset G$ and a closed subgroup $H$ of $G$, any orbit closure $\overline{H x}, x \in G / \Gamma$, is an orbit of an intermediate subgroup $L \supset H$ of $G$ unless "it has a good reason not to" (the latter reasons must be coming from certain one-parameter quotient actions). See [Ma8, $\S 1]$ for more detail.

Multiplicative approximation on manifolds. Since every VWA vector is VWMA (that is, $\psi_{\varepsilon}$-MA for some $\varepsilon>0$ ) but not other way around, it is a more difficult problem to prove that a generic point on a nondegenerate manifold is not very well multiplicatively approximable. This has been known as Conjecture $\mathrm{H}_{2}$ of Sprindžuk [Sp4]; the polynomial special case (that is, a multiplicative strengthening of Mahler's problem) was conjectured by Baker in [B2]; both conjectures stood open, except for low-dimensional special cases, until [KM2] where the following was proved: 
Theorem 5.2. Let $M$ be a nondegenerate smooth submanifold of $\mathbb{R}^{n}$. Then almost all points of $M$ are not VWMA.

The strategy of the proof of Theorem 4.1 applies with minor changes. One shows (see [KM2, Lemma 2.1 and Corollary 2.2] for a partial result) that $\mathbf{y} \in \mathbb{R}^{n}$ is VWMA iff for some $\gamma>0$ there are infinitely many $\mathbf{t} \in \mathbb{Z}_{+}^{n}$ such that

$$
g_{\mathbf{t}} L_{\mathbf{y}} \mathbb{Z}^{k} \in \Omega_{e^{-\gamma t}}
$$

(here, as defined above, $t$ stands for $\sum_{i=1}^{n} t_{i}$ ). Therefore it is enough to use Theorem 4.6 to prove a modification of the measure estimate of Theorem 4.4 with $g_{t}$ as in (4.5) replaced by $g_{\mathbf{t}}$ as in (5.1).

Finally let us mention a multiplicative version of Theorem 4.2, proved in [BKM] by a modification of the method described above:

Theorem 5.3. Let $M$ be a nondegenerate smooth submanifold of $\mathbb{R}^{n}$ and let $\psi$ be such that $\sum_{l=1}^{\infty}(\log l)^{n-1} \psi(l)$ is finite. Then almost all points of $M$ are not $\psi$-multiplicatively approximable.

\section{ACKNOWLEDGEMENTS}

This survey is based on a minicourse of lectures given at the AMS Summer Research Institute in Smooth Ergodic Theory and applications (Seattle, 1999). The author is grateful to the organizers and participants of the workshop, and especially to Alex Eskin for sharing the responsibility and the fun of giving the course. Thanks are also due to participants/organizers of the workshop on Ergodic Theory, Rigidity and Number Theory (Cambridge UK, January 2000) where the presentation of material was tested one more time in a series of lectures, to Gregory Margulis, Alexander Starkov and Barak Weiss for helpful discussions, and to Stella for her endless patience and support.

\section{REFERENCES}

[AGH] L. Auslander, L. Green and F. Hahn, Flows on homogeneous spaces, Annals of Mathematics Studies, No. 53, Princeton University Press, Princeton, N.J., 1963.

[AN1] A. G. Abercrombie and R. Nair, An exceptional set in the ergodic theory of Markov maps of the interval, Proc. London Math. Soc. 75 (1997), 221-240.

[AN2] - An exceptional set in the ergodic theory of rational maps of the Riemann sphere, Ergodic Theory Dynam. Systems 17 (1997), 253-267.

[B1] A. Baker, On a theorem of Sprindzhuk, Proc. Roy. Soc. London A 292 (1966), 92-104.

[B2] , Transcendental number theory, Cambridge Univ. Press, Cambridge, 1975.

[Bek] M. Bekka, On uniqueness of invariant means, Proc. Amer. Math. Soc. 126 (1998), 507514.

[Bere1] V. Beresnevich, On approximation of real numbers by real algebraic numbers, Acta Arith. 90 (1999), 97-112.

[Bere2] — A Groshev type theorem for convergence on manifolds, Acta Math. Hungar. (to appear).

[Bern] V. Bernik, A proof of Baker's conjecture in the metric theory of transcendental numbers, Doklady Akad. Nauk SSSR 277 (1984), 1036-1039. (Russian)

[BD] V. Bernik and M. M. Dodson, Metric Diophantine approximation on manifolds, Cambridge Univ. Press, Cambridge, 1999.

[BKM] V. Bernik, D. Kleinbock and G. A. Margulis, Khintchine-type theorems on manifolds: the convergence case for standard and multiplicative versions, Preprint (2000).

[BM] J. Brezin and C. C. Moore, Flows on homogeneous spaces: a new look, Amer. J. Math. 103 (1981), 571-613. 
[BMa] B. Bekka and M. Mayer, Ergodic theory and topological dynamics of group actions on homogeneous spaces, Cambridge University Press, Cambridge, 2000.

[C] J. W. S. Cassels, An introduction to Diophantine approximation, Cambridge Tracts in Math., vol. 45, Cambridge Univ. Press, Cambridge, 1957.

[CK] N. Chernov and D. Kleinbock, Dynamical Borel-Cantelli lemmas for Gibbs measures, Israel J. Math. (to appear).

[CR] J.-P. Conze and A. Raugi, Convergence des potentiels pour un opérateur de transfert, applications aux systèmes dynamiques et aux chaînes de Markov, Preprint (1999).

[CS] J.W.S. Cassels and H.P.F. Swinnerton-Dyer, On the product of three homogeneous forms and indefinite ternary quadratic forms, Philos. Trans. Roy. Soc. London 248, Ser. A (1955), 73-96.

[D1] S. G. Dani, Divergent trajectories of flows on homogeneous spaces and Diophantine approximation, J. Reine Angew. Math. 359 (1985), 55-89.

[D2] , Bounded orbits of flows on homogeneous spaces, Comment. Math. Helv. 61 (1986), 636-660.

[D3] On orbits of unipotent flows on homogeneous spaces, II, Ergodic Theory Dynamical Systems 6 (1986), 167-182.

[D4] , On orbits of endomorphisms of tori and the Schmidt game, Ergodic Theory Dynam. Systems 8 (1988), 523-529.

[D5] - Flows on homogeneous spaces and Diophantine approximation, Proceedings of the International Congress of Mathematicians, Vol. 1, 2 (Zürich, 1994), Birkhäuser, Basel, 1995, pp. 780-789.

[D6] Flows on homogeneous spaces: a review, Ergodic theory of $Z^{d}$ actions (Warwick, 1993-1994), Cambridge Univ. Press, Cambridge, 1996, pp. 63-112.

[D7] Dynamical systems on homogeneous spaces, in: Dynamical systems, ergodic theory and applications,, Springer-Verlag, Berlin, 2000.

[Dod] M. M. Dodson, Geometric and probabilistic ideas in metric Diophantine approximation, Russian Math. Surveys 48 (1993), 73-102.

[Dol1] D. Dolgopyat, Bounded orbits of Anosov flows, Duke Math. J 87 (1997), 87-114.

[Dol2] - Limit theorems for partially hyperbolic systems, Preprint (1998).

[DM1] S.G. Dani and G.A. Margulis, Values of quadratic forms at primitive integral points, Invent. Math. 98 (1989), 405-424.

[DM2] - Limit distributions of orbits of unipotent flows and values of quadratic forms, Adv. in Soviet Math., vol. 16, Amer. Math. Soc., Providence, R.I., 1993, pp. 91-137.

[E] A. Eskin, Counting problems and semisimple groups, Proceedings of the International Congress of Mathematicians, Vol. II (Berlin, 1998), Doc. Math., 1998, pp. 539-552.

[G] P. Gallagher, Metric simultaneous diophantine approximation, J. London Math. Soc. 37 (1962), 387-390.

[Gr] A. V. Groshev, Une théorème sur les systèmes des formes linéaires, Dokl. Akad. Nauk SSSR 9 (1938), 151-152.

[EMM] A. Eskin, G. A. Margulis and S. Mozes, Upper bounds and asymptotics in a quantitative version of the Oppenheim conjecture, Ann. Math. 147 (1998), 93-141.

[EMS] A. Eskin, S. Mozes and N. Shah, Non-divergence of translates of certain algebraic measures, Geom. Funct. Anal. 7 (1997), 48-80.

[H] G. Harman, Metric number theory, London Mathematical Society Monographs, New Series, vol. 18, The Clarendon Press, Oxford University Press, New York, 1998.

[K1] D. Kleinbock, Nondense orbits of flows on homogeneous spaces, Ergodic Theory Dynamical Systems 18 (1998), 373-396.

[K2] Flows on homogeneous spaces and Diophantine properties of matrices, Duke Math. J. 95 (1998), 107-124.

[K3] , Bounded orbit conjecture and diophantine approximation, in: Proceedings of the International Colloquium on Lie Groups and Ergodic Theory, TIFR, Mumbai, 1998, pp. 119-130.

[K4] , Badly approximable systems of affine forms, J. Number Theory 79 (1999), 83102.

[KM1] D. Kleinbock and G. A. Margulis, Bounded orbits of nonquasiunipotent flows on homogeneous spaces, Amer. Math. Soc. Transl. 171 (1996), 141-172. 
[KM2] - Flows on homogeneous spaces and Diophantine approximation on manifolds, Ann. Math. 148 (1998), 339-360.

[KM3] - Logarithm laws for flows on homogeneous spaces, Inv. Math. 138 (1999), 451494.

[KS] A. Katok and R. Spatzier, First cohomology of Anosov actions of higher rank Abelian groups and applications to rigidity, Inst. Hautes Études Sci. Publ. Math. 79 (1994), 131156.

[KSS] D. Kleinbock, N. Shah, and A. Starkov, Homogeneous flows, applications to number theory, and related topics, in: Handbook on Dynamical Systems (Encyclopedia of Math Sciences Series), Elsevier (to appear).

[LW] E. Lindenstrauss and B. Weiss, On sets invariant under the action of the diagonal group, SUNY Stony Brook IMS Preprint \# 1999/8.

[M] K. Mahler, Über das Mass der Menge aller S-Zahlen, Math. Ann. 106 (1932), 131-139.

[Ma1] G. A. Margulis, On some problems in the theory of U-systems (in Russian), Thesis, Moscow University, 1970.

[Ma2] - On the action of unipotent group in the space of lattices, Proceedings of the Summer School on group representations, (Budapest 1971), Académiai Kiado, Budapest, 1975, pp. 365-370.

[Ma3] - Formes quadratriques indéfinies et flots unipotents sur les espaces homogénes. C. R. Acad. Sci. Paris Sér. I Math. 304 (1987), 249-253.

[Ma4] Discrete subgroups and ergodic theory, Proc. of the conference "Number theory, trace formulas and discrete groups" in honour of A. Selberg (Oslo, 1987), Academic Press, Boston, MA 1989, 377-398.

[Ma5] - Dynamical and ergodic properties of subgroup actions on homogeneous spaces with applications to number theory, Proceedings of the International Congress of Mathematicians, Vol. I, II (Kyoto, 1990), Math. Soc. Japan, Tokyo, 1991, pp. 193-215.

[Ma6] _ Discrete subgroups of semisimple Lie groups, Springer-Verlag, Berlin and New York, 1991.

[Ma7] , Oppenheim conjecture, Fields Medallists' lectures, World Sci. Publishing, River Edge, N.J., 1997, pp. 272-327.

[Ma8] - Problems and conjectures in rigidity theory, in: Mathematics: Frontiers and Perspectives, Amer. Math. Soc., Providence, R.I., 2000.

[Mo1] C. C. Moore, Ergodicity of flows on homogeneous spaces, Amer. J. Math. 88 (1966), $154-178$

[Mo2] _ Exponential decay of correlation coefficients for geodesic flows, Group Representations, Ergodic Theory, Operator Algebras and Mathematical Physics, Math. Sci. Res. Inst. Publ., vol. 6, Springer-Verlag, Berlin and New York, 1987, pp. 163-181.

[Op1] A. Oppenheim, The minima of indefinite quaternary quadratic forms, Proc. Nat. Acad. Sci. USA 15 (1929), 724-727.

[Op2] Values of quadratic forms I, Quart. J. Math. Oxford Ser. (2) 4 (1953), 54-59.

$[\mathrm{Ph}] \quad$ W. Philipp, Some metrical theorems in number theory, Pacific J. Math. 20 (1967), 109127.

[R] M. S. Raghunathan, Discrete subgroups of Lie groups, Springer-Verlag, Berlin and New York, 1972.

[Ra1] M. Ratner, The rate of mixing for geodesic and horocycle flows, Ergodic Theory Dynamical Systems 7 (1987), 267-288.

[Ra2] - Raghunathan's topological conjecture and distribution of unipotent flows, Duke Math. J. 63 (1991), 235-280.

[Ra3] - Interactions between ergodic theory, Lie groups, and number theory, Proceedings of the International Congress of Mathematicians, Vol. 1, 2 (Zürich, 1994), Birkhäuser, Basel, 1995, pp. 157-182.

[S1] W. M. Schmidt, A metrical theorem in Diophantine approximation, Canadian J. Math. 12 (1960), 619-631.

[S2] _ Badly approximable systems of linear forms, J. Number Theory 1 (1969), 139154.

[S3] Diophantine approximation, Springer-Verlag, Berlin and New York, 1980

[Sh] N. Shah, Limit distribution of polynomial trajectories on homogeneous spaces, Duke Math. J. 75 (1994), 711-732. 
[Sp1] V. Sprindžuk, More on Mahler's conjecture, Doklady Akad. Nauk SSSR 155 (1964), 54-56 (Russian); English transl. in Soviet Math. Dokl 5 (1964), 361-363.

[Sp2] V. Sprindžuk, Mahler's problem in metric number theory, Translations of Mathematical Monographs, vol. 25, Amer. Math. Soc., Providence, R.I., 1969.

[Sp3] _ Metric theory of Diophantine approximations, John Wiley \& Sons, New YorkToronto-London, 1979.

[Sp4] - Achievements and problems in Diophantine approximation theory, Russian Math. Surveys 35 (1980), 1-80.

[St1] A. N. Starkov, New progress in the theory of homogeneous flows, Russian Math. Surveys 52 (1997), 721-818.

[St2] Dynamical systems in homogeneous spaces, Translations of Mathematical Monographs, vol. 190, Amer. Math. Soc., Providence, R.I., 2000.

[Su] D. Sullivan, Disjoint spheres, approximation by imaginary quadratic numbers, and the logarithm law for geodesics, Acta Math. 149 (1982), 215-237.

[U] M. Urbanski, The Hausdorff dimension of the set of points with nondense orbit under a hyperbolic dynamical system, Nonlinearity 2 (1991), 385-397.

[Z] R. Zimmer, Ergodic theory and semisimple groups, Birkhäuser, Boston, 1984.

Dmitry Kleinbock, Department of Mathematics, Brandeis University, Waltham, MA 02454-9110

E-mail address: kleinboc@brandeis.edu 